OPEN ACCESS

Edited by:

Paulo Arruda,

Universidade Estadual de Campinas,

Brazil

Reviewed by:

Jianhua Fan,

Carnegie Institution for Science (CIS),

United States

Jian Li Yang,

Zhejiang University, China

Tamás Papp,

University of Szeged, Hungary

Taras P. Pasternak,

Albert Ludwigs University of Freiburg,

Germany

${ }^{*}$ Correspondence:

Jianhua Wang

wangjh63@cau.edu.cn

Yunjun Liu

liuyunjun@caas.cn

Specialty section:

This article was submitted to

Plant Biotechnology,

a section of the journal

Frontiers in Plant Science

Received: 17 May 2017 Accepted: 29 September 2017 Published: 17 October 2017

Citation:

Liu Y, Zhang Z, Fu J, Wang G, Wang J and Liu Y (2017) Transcriptome Analysis of Maize Immature Embryos

Reveals the Roles of Cysteine in Improving Agrobacterium Infection Efficiency. Front. Plant Sci. 8:1778. doi: $10.3389 /$ fpls.2017.01778

\section{Transcriptome Analysis of Maize Immature Embryos Reveals the Roles of Cysteine in Improving Agrobacterium Infection Efficiency}

\author{
Yan Liu ${ }^{1,2}$, Zhiqiang Zhang ${ }^{1,2}$, Junjie $\mathrm{Fu}^{2}$, Guoying Wang ${ }^{2}$, Jianhua Wang $^{1 *}$ and \\ Yunjun Liu $^{2 *}$ \\ ${ }^{1}$ College of Agronomy and Biotechnology, China Agricultural University, Beijing, China, ${ }^{2}$ Institute of Crop Sciences, Chinese \\ Academy of Agricultural Sciences, Beijing, China
}

Maize Agrobacterium-mediated transformation efficiency has been greatly improved in recent years. Antioxidants, such as, cysteine, can significantly improve maize transformation frequency through improving the Agrobacterium infection efficiency. However, the mechanism underlying the transformation improvement after cysteine exposure has not been elucidated. In this study, we showed that the addition of cysteine to the co-cultivation medium significantly increased the Agrobacterium infection efficiency of hybrid Hill and inbred line Z31 maize embryos. Reactive oxygen species contents were higher in embryos treated with cysteine than that without cysteine. We further investigated the mechanism behind cysteine-related infection efficiency increase using transcriptome analysis. The results showed that the cysteine treatment upregulated 939 genes and down-regulated 549 genes in both Z31 and Hill. Additionally, more differentially expressed genes were found in Hill embryos than those in Z31 embryos, suggesting that Hill was more sensitive to the cysteine treatment than Z31. GO analysis showed that the up-regulated genes were mainly involved in the oxidation reduction process. The up-regulation of these genes could help maize embryos to cope with the oxidative stress stimulated by Agrobacterium infection. The down-regulated genes were mainly involved in the cell wall and membrane metabolism, such as, aquaporin and expansin genes. Decreased expression of these cell wall integrity genes could loosen the cell wall, thereby improving the entry of Agrobacterium into plant cells. This study offers insight into the role of cysteine in improving Agrobacterium-mediated transformation of maize immature embryos.

\section{Keywords: Agrobacterium, infection efficiency, cysteine, maize embryo, transcriptome}

\section{INTRODUCTION}

An efficient crop transformation method is one of the prerequisites for creating transgenic crops. At present, widely used transformation methods include the particle bombardment method and the Agrobacterium-mediated method. Compared with particle bombardment, Agrobacterium-mediated transformation has the advantages of simple operation, low cost, low foreign gene copy number, and the stable inheritance of foreign genes. Through whole genome 
mutation and phenotype screening, many Agrobacterium genes were identified that play key roles in the transformation process. One collection of genes involved in excising DNA and integrating it into a foreign genome is the Vir region. It is well known that phenols or jasmonic acid can induce the expression of Agrobacterium Vir genes under the acidic environments. The transcriptome and proteome analysis of Agrobacterium treated with acetosyringone, illustrated that Vir proteins have different expression response mechanisms (Cho and Winans, 2005; Lai et al., 2006). It is known that $\operatorname{Vir} A$ and $\operatorname{Vir} G$ maintain constant expression levels in Agrobacterium. When stimulated with an outside signal, the protein kinase VirA can phosphorylate VirG and increase its expression; As a transcription factor, VirG proteins activate the expression of other Vir genes (Brencic and Winans, 2005). The T-complexes formed between VirD2 and T-DNA enter the plant cell, where the T-complexes can now bind the other Vir proteins (VirE2, VirE3, VirD5, and VirF) to form a super complex. This super complex can enter the plant cell nucleus and release the T-DNA for integration into the plant genome (Gelvin, 2010; Pitzschke and Hirt, 2010).

Plant genes also participate in the process of Agrobacterium mediated transformation (Pitzschke and Hirt, 2010). Through screening mutants resistant to Agrobacterium tumefaciens transformation, more than 129 genes have been identified to influence the Arabidopsis transformation by A. tumefaciens (Zhu et al., 2003b). In tobacco cells, the Agrobacterium VirE2 proteins have been shown to interact with tobacco VIP1 and VIP2 proteins, and VIP1 overexpression significantly improved the transformation efficiency (Tzfira et al., 2002). It was shown that VIP1 is a substrate of the MPKK signaling pathway in the plant, where signals are transferred from the cytoplasm to the nucleus after phosphorylation by MPKKK, mediating the entry of the Vir protein and T-DNA T-complex (Djamei et al., 2007). The plant genes AtAGP17, encoding a galactosan (Gaspar et al., 2004) and CslA-09 gene, encoding cellulose synthase (Zhu et al., 2003a), play roles in Agrobacterium attachment to plant cells. The plant transporter $\beta 3$ has been shown to play an important role in the entry of Vir and T-DNA into the nucleus (Zhu et al., 2003b). On the contrary, the Arabidopsis MTF1 protein has been shown to negatively affect the attachment of several Agrobacterium strains to roots, thereby decreasing the transformation efficiency (Sardesai et al., 2014). Other plant proteins, such as, SUPPRESSOR OF G2 ALLELE OF SKP1 (SGT1) and heat shock protein 90.1, are required for Agrobacterium-mediated transformation (Anand et al., 2012; Park et al., 2014). Many plant genes play important roles in the controlling of callus formation and organ re-differentiation, including AUXIN RESPONSE FACTORs, LEAFY COTYLEDON1, WUSCHEL, BABY BOOM, AGAMOUS-LIKE15, and SOMATIC EMBRYOGENESIS RECEPTOR KINASE (Altpeter et al., 2016). Overexpression of maize Baby boom (Bbm) and maize Wuschel2 (Wus2) genes have been shown to achieve high transformation efficiency in numerous recalcitrant maize inbred lines (Lowe et al., 2016; Mookkan et al., 2017).

To achieve high efficient Agrobacterium-mediated maize transformation, the plant genotypes, explants, Agrobacterium strains, co-cultivation medium and other factors should be taken into consideration. The milepost type of work on Agrobacterium-mediated maize transformation comes from Ishida et al. (1996), where a super binary vector carrying the $\operatorname{vir} B$, virC, and $\operatorname{virG}$ genes, was used to infect the immature embryos of maize inbred line A188, achieving 5-30\% transformation efficiency. Maize transformation efficiency has also been improved by treating immature embryos with heat prior to Agrobacterium infection (Hiei et al., 2006). In aforementioned maize transformation systems, in vitro tissue culture was maintained in dark conditions. Cho et al. (2014) established a high frequency maize transformation protocol for recalcitrant maize inbred lines, by initiating and maintaining green tissues under dim light condition.

Antioxidants such as, cysteine, dithiothreitol (DTT), glutathione, and ascorbic acid can reduce tissue necrosis in explants during plant transformation. Cysteine is often used to improve the transformation efficiency of soybean (Olhoft and Somers, 2001; Zeng et al., 2004). In sugarcane transformation, usage of the media containing ascorbic acid and cysteine has been shown to offer higher transformation efficiency (Enríquez-Obregón et al., 1998). It has also been shown that the anti-oxidative compounds lipoic acid enhances the Agrobacterium-mediated transformation of MicroTom and soybean by mitigating oxidative stress (Dan et al., 2015). The addition of cysteine or DTT in co-cultivation medium has also been shown to significantly improve maize transformation efficiency (Frame et al., 2002; Vega et al., 2008).

Although it has been shown that cysteine can improve the transformation efficiency, the mechanism has not been elucidated. In this study, we showed that cysteine could improve Agrobacterium infection efficiency in the maize inbred line Z31 and hybrid line HiII. We further investigated the mechanism behind cysteine-related infection efficiency increase using transcriptome analysis. The results showed that the up-regulated genes were mainly involved in the oxidation reduction process, and the down-regulated genes were mainly involved in the cell wall and membrane metabolism. We hypothesize that cysteine loosens the cell wall by modifying the expression of genes involved in cell wall metabolism and membrane metabolism, i.e., aquaporin and expansin genes, thereby improving the entry of Agrobacterium into plant cells.

\section{MATERIALS AND METHODS}

\section{Plant Material and Agrobacterium Strain}

Inbred line Z31 and hybrid line Hill were used in this study. Maize plants were grown in the greenhouse under a $16 / 8 \mathrm{~h}$ light/dark cycle at $20-25^{\circ} \mathrm{C}$. The Agrobacterium strain used in this study was EHA105 containing the binary vector pCambia3301 that carries a T-DNA with a beta-glucuronidase (gus) gene.

\section{Immature Embryo Treatment}

The immature embryos were collected from the maize ears 10-12 days after pollination. The ears were immersed in filter-sterilized MS-INF (4.1 $\mathrm{g} \mathrm{L}^{-1}$ MS medium, $0.115 \mathrm{~g} \mathrm{~L}^{-1}$ L-Proline, $36 \mathrm{~g} \mathrm{~L}^{-1}$ glucose, $68.5 \mathrm{~g} \mathrm{~L}^{-1}$ sucrose, $100 \mu \mathrm{M}$ 
acetosyringone, $\mathrm{pH}$ 5.4). The Agrobacterium strain was cultured in autoclaved liquid YEP medium $\left(10 \mathrm{~g} \mathrm{~L}^{-1}\right.$ tryptone, $10 \mathrm{~g}$ $\mathrm{L}^{-1}$ yeast extract, $5 \mathrm{~g} \mathrm{~L}^{-1} \mathrm{NaCl}, 50 \mathrm{mg} \mathrm{L}^{-1}$ rifampicin, $50 \mathrm{mg}$ $\mathrm{L}^{-1}$ kanamycin, $\mathrm{pH} 7.0$ ) overnight shaking at $220 \mathrm{rpm}$ at $28^{\circ} \mathrm{C}$. The Agrobacterium was collected by centrifuge and resuspended to $\mathrm{OD}_{600}=0.4$ in the MS-INF medium. The collected embryos were heated in a $45^{\circ} \mathrm{C}$ water bath for $2 \mathrm{~min}$, and then infected with the Agrobacterium suspension for $5 \mathrm{~min}$. After infection, the immature embryos were transferred onto MS COmedium $\left(4.1 \mathrm{~g} \mathrm{~L}^{-1} \mathrm{MS}\right.$ medium, $0.7 \mathrm{~g} \mathrm{~L}^{-1} \mathrm{~L}$-Proline, $10 \mathrm{~g} \mathrm{~L}^{-1}$ glucose, $20 \mathrm{~g} \mathrm{~L}^{-1}$ sucrose, $0.85 \mathrm{mg} \mathrm{L}^{-1}$ silver nitrate, $100 \mu \mathrm{M}$ acetosyringone, $1.5 \mathrm{mg} \mathrm{mL}^{-1} 2,4-\mathrm{D}, 3.5 \mathrm{~g} \mathrm{~L}^{-1}$ phytagel, $\mathrm{pH}$ 5.8) with or without $100 \mathrm{mg} \mathrm{L}^{-1}$ cysteine that was freshly prepared and filter-sterilized. After culturing for 3 days at $25^{\circ} \mathrm{C}$ under dark conditions, the immature embryos were collected, and stored at $-70^{\circ} \mathrm{C}$ for RNA extraction. Ultimately, four treatments were applied to the maize embryos: (1) no heat and no cysteine-infused media, (2) no heat and cysteine-infused media, (3) heat and no cysteine-infused media, and (4) heat and cysteine-infused media.

\section{Analysis of Transient GUS Expression}

Three days after growth on media with or without cysteine, the transient gus expression was analyzed using a histochemical GUS assay. The embryos were immersed in the GUS staining solution (Jefferson et al., 1987) and incubated overnight at $37^{\circ} \mathrm{C}$. The level of transient gus expression was assessed based on the number of visible blue foci on the scutellum side of each embryo (Frame et al., 2002).

\section{Reactive Oxygen Species Determination}

The Z31 immature embryos were cultured for 3 days at $25^{\circ} \mathrm{C}$ in dark condition on media with or without cysteine after infection by A. tumefaciens EHA105. The immature embryos $(0.1 \mathrm{~g})$ were homogenized with phosphate buffer saline (PBS) solution $(\mathrm{pH}$ 7.4), and then centrifuged at $8,000 \mathrm{rpm}$ and $4^{\circ} \mathrm{C}$ for $30 \mathrm{~min}$, and the supernatant was used for subsequent reactions. The hydrogen peroxide $\left(\mathrm{H}_{2} \mathrm{O}_{2}\right)$ content was measured using an enzyme-linked immunosorbent assay (ELISA) kit (Tsz Biosciences, Woburn, USA). The superoxide anion content was measured using an ELISA kit (SU-B91178, Kenuodi Biotech, Fujian, China). In situ superoxide anion $\left(\mathrm{O}_{2}^{-}\right)$was estimated using the NBT staining method and $\mathrm{H}_{2} \mathrm{O}_{2}$ using the DAB staining method (Dutilleul et al., 2003).

\section{Transcriptome Analysis}

Total RNA was extracted from embryos after 3-day co-cultivation with or without cysteine using the RNeasy Plant Mini Kit (Qiagen, Germany). The extracted RNA was run in a 1\% agarose gel to assess the integrity of the RNA. The RNA yield and purity were checked using the Nano-drop ND-1000. Poly(A) mRNAs were isolated from the total RNA using oligo ( $\mathrm{dT}$ ) magnetic beads (Illumina, San Diego, CA). RNA fragmentation, cDNA synthesis, and PCR amplification were performed according to the Illumina RNA-Seq protocol. The cDNA libraries were sequenced with a read length of $100 \mathrm{bp}$ (paired-end) using the Illumina HiSeq 2000 System at Berry Genomics (Beijing, China). The experiment was performed with four biological replicates.

\section{Data Analysis}

The obtained raw data were processed with Perl scripts to remove the adaptor-polluted reads, low-quality reads and reads with the number of $\mathrm{N}$ bases accounting for more than 5\%. The filtered reads were used for the quality and quantity analyses. The TopHat software v2.0.12 was used to map the clean reads to the B73 RefGen_V3 genome (www.maizegdb.org).

FPKM (fragments per kilobase of transcripts per million fragments mapped) was calculated to estimate the expression level of each sample. The Cuffdiff program within the Cufflinks software was used to identify the differentially expressed genes (DEGs) based on the following thresholds: $\mid$ fold change $\mid \geq 2$ and FDR cut-off $<0.01$.

MapMan software (version 3.5.1R2; http://mapman.gabipd. org/) was used to analyze the metabolic pathways according to the top hits to the maize genome database. In addition, agriGO (http://bioinfo.cau.edu.cn/agriGO/) was used to estimate the biological process, molecular function, and cellular component for each DEGs.

\section{Quantitative Real-Time PCR Analysis}

The first-strand cDNA synthesis was performed with the M-MuLV reverse transcriptase (Promega) using total RNA as the template. For the quantitative real-time PCR (qRT-PCR), 1 $\mu \mathrm{L}$ of cDNA was mixed with $2 \times$ SYBR premix ExTaq (Takara), $0.2 \mu \mathrm{M}$ forward primer, $0.2 \mu \mathrm{M}$ reverse primer, and $0.4 \mu \mathrm{L} 50 \times$ ROX in $20 \mu \mathrm{L}$ of reaction mixture. The qRT-PCR was conducted using the ABI 7300 system with the following protocol: $95^{\circ} \mathrm{C}$ for $2 \mathrm{~min}, 40 \mathrm{cycles}$ at $95^{\circ} \mathrm{C}$ for $5 \mathrm{~s}, 58^{\circ} \mathrm{C}$ for $30 \mathrm{~s}$, and $72^{\circ} \mathrm{C}$ for $31 \mathrm{~s}$. The relative transcriptional levels were calculated using the $2^{-\Delta \Delta \mathrm{Ct}}$ method (Livak and Schmittgen, 2001) with actin as a housekeeping gene.

\section{RESULTS}

\section{Heat and Cysteine Treatments Improve the Agrobacterium Infection Efficiency of Maize Embryos}

It has been reported that the addition of cysteine in medium can significantly improve transformation efficiency in the maize hybrid HiII (Frame et al., 2002; Vega et al., 2008). Heat shock is also an efficient way to improve the transformation efficiency (Hiei et al., 2006). To test the transformation efficiency in the maize elite inbred line Z31 and maize hybrid HiII, we combined both strategies, including an embryo heat treatment prior to Agrobacterium infection and the addition of cysteine in the cocultivation medium. The Agrobacterium strain EHA105 carrying the binary vector pCambia3301 was used for maize embryo transformation with the gus gene. After culturing with or without cysteine for 3 days, the transient GUS expression was analyzed by histochemical GUS assays. For both Z31 and HiII materials, heat treatment somewhat increased infection efficiency. Compared with heat treatment, the addition of cysteine in the co-cultivation medium had greater effects on the infection efficiency (Figures 1, 2). High infection efficiency was observed for the HiII material, compared to maize inbred line Z31 (Figures 1, 2). 


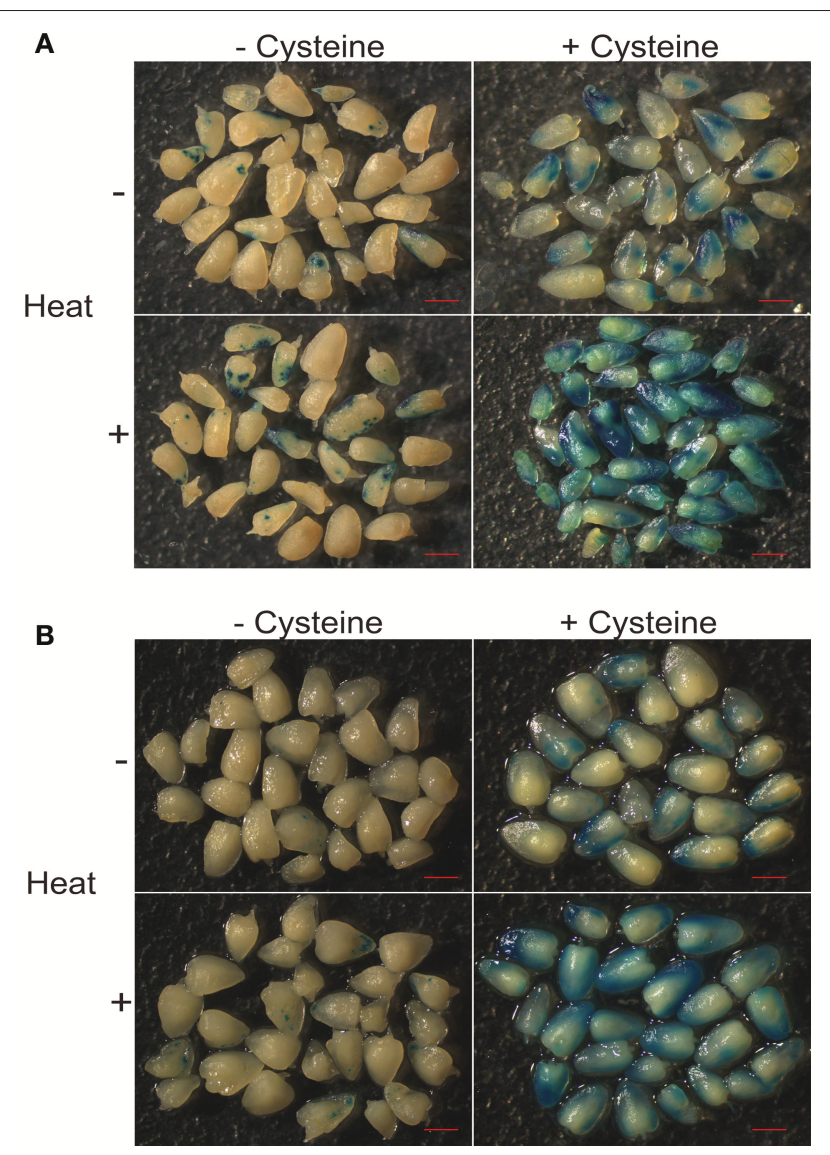

FIGURE 1 | Heat treatment and cysteine effect on Agrobacterium infection efficiency. (A) Maize hybrid Hill; (B) Maize inbred line Z31.

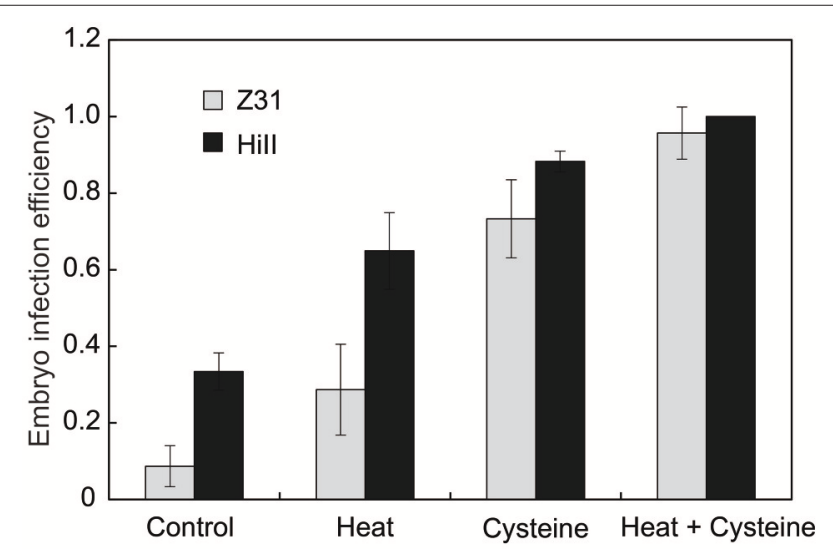

FIGURE 2 | The embryo infection efficiency. Data was shown as the average \pm s.e. of three independent experiments.

\section{Reactive Oxygen Species Accumulation in Agrobacterium-Infected Embryos}

The reactive oxygen species hydrogen peroxide $\left(\mathrm{H}_{2} \mathrm{O}_{2}\right)$ and superoxide anion $\left(\mathrm{O}_{2}^{-}\right)$levels were measured in the
Agrobacterium-infected embryos. Agrobacterium infection stimulated the accumulation of $\mathrm{H}_{2} \mathrm{O}_{2}$ in embryos grown on co-cultivation medium with or without cysteine (Figures $3 \mathrm{~A}, \mathrm{C}$ ), whereas did not affect the accumulation of $\mathrm{O}_{2}^{-}$(Figures 3B,D). To our surprise, the addition of cysteine in the co-cultivation medium significantly increased the accumulation of $\mathrm{H}_{2} \mathrm{O}_{2}$ and $\mathrm{O}_{2}^{-}$in embryos (Figure 3). These results indicated that cysteine may improve the Agrobacterium infection efficiency not just as an antioxidant, but with other mechanism.

\section{Maize Embryo Transcriptome Profiling}

To investigate the mechanism of cysteine-related transformation efficiency improvement, we performed transcriptome analysis of the maize embryos cultured on the medium with or without cysteine. The experiment was performed with four independent biological replicates. We obtained 7,432,161-15,904,122 reads from the HiII embryo samples and more than $78.73 \%$ of the reads were mapped to the $\mathrm{B} 73$ reference genome. We obtained 20,119,176-24,789,278 reads from the inbred line Z31 embryo samples and $73.66 \%$ of the reads were mapped to the $\mathrm{B} 73$ reference genome (Table 1). Maize has approximately 30,000 genes and the sequencing depth we acquired was enough for subsequent analysis.

In this study, $\mid$ fold change $\mid \geq 2$ and FDR cut-off $<0.01$ was set as the threshold to select for DEGs. A total of 2,795 and 1,277 genes were up-regulated in the cysteine treatment in Z31 and HiII, respectively. Among them, 939 genes were up-regulated in both Z31 and HiII. A total of 2,150 and 949 genes were down-regulated in the cysteine treatment in Z31 and HiII, respectively. Five hundred and forty-nine genes among them were down-regulated in both Z31 and HiII (Figure 4). To confirm the accuracy of the transcriptome analysis results, the transcripts of 11 genes were analyzed using qRT-PCR. The primers for these genes are shown in Table S1. The qRT-PCR results showed that the expression profiles of these genes were consistent with the RNA sequencing data (Table 2), indicating that our RNA sequencing results were valid.

\section{Metabolism Overview of Differentially Expressed Genes in Maize Embryos}

To elucidate how cysteine influences Agrobacterium infection efficiency, the putative function of DEGs was analyzed using Blast2GO. We performed GO analysis of the up-regulated and down-regulated genes in HiII and Z31. Due to fact that HiII and Z31 had different responses to the cysteine treatment, the common DEGs in both materials were used to elucidate the mechanism. The common up-regulated genes were divided into the following categories: metabolic process, oxidation-reduction process, L-phenylalanine catabolic process, transferase activity, transferring hexosyl groups, oxidoreductase activity, ammonialyase activity, iron ion binding, and catalytic activity (Figure 5). Results showed that $24 \%$ of the common up-regulated genes belonged to the oxidation-reduction process and $16 \%$ of the genes belonged to the oxidoreductase activity process. These two processes have important roles in the regulation of the redox balance of the plant cells. As a reducing agent, cysteine may induce the expression of the redox genes and lead to high 
A

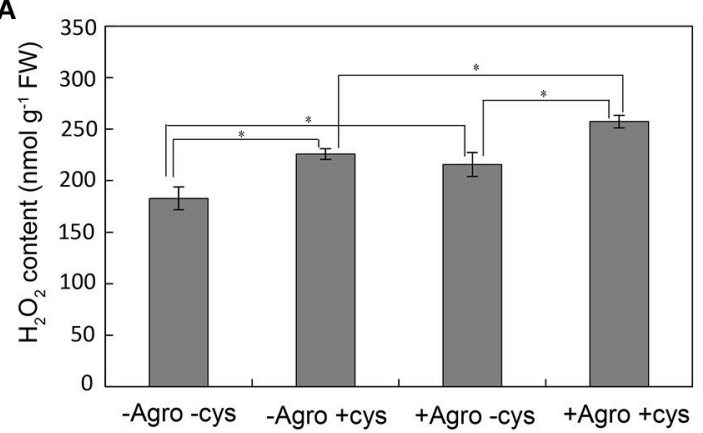

C
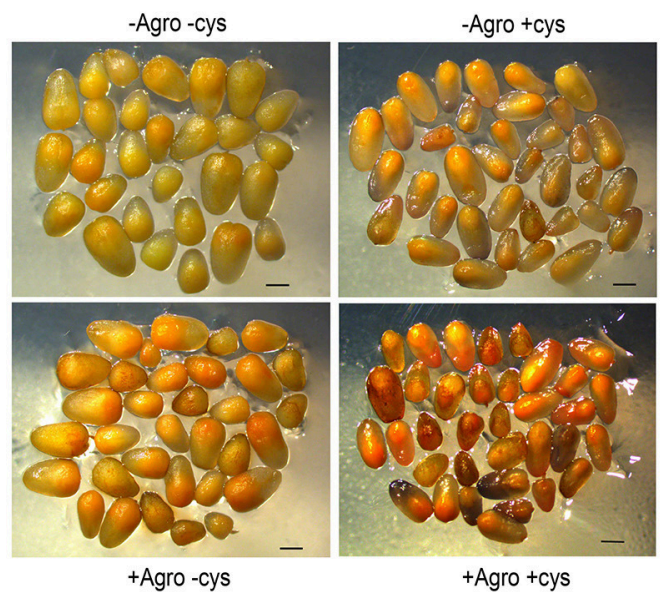

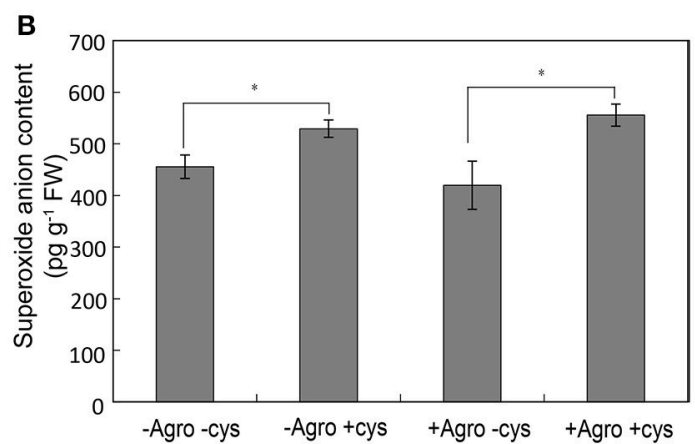

D

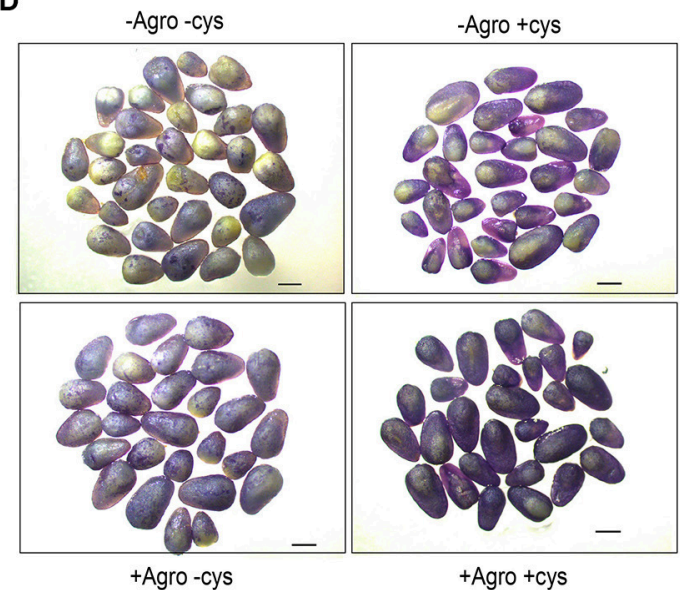

FIGURE 3 | Reactive oxygen species contents in maize embryos. The Z31 immature embryos were infected or not infected by Agrobacterium tumefaciens EHA105, then cultured for 3 days at $25^{\circ} \mathrm{C}$ in dark condition on co-cultivation media with or without cysteine. $\mathrm{H}_{2} \mathrm{O}_{2}$ (A) and $\mathrm{O}_{2}^{-}$(B) contents in embryos were measured and the data was shown as average \pm s.e. of three independent experiments. Asterisks indicated the significant difference at $P<0.05$ level. In situ $\mathrm{H}_{2} \mathrm{O}_{2}$ (C) and $\mathrm{O}_{2}^{-}$(D) was estimated using the DAB and NBT staining method, respectively.

TABLE 1 | RNA-seq reads of maize embryo mapped to the maize B73 RefGen_V3 genome.

\begin{tabular}{llcccc}
\hline $\begin{array}{l}\text { Maize } \\
\text { lines }\end{array}$ & Sample & Repeat & Total reads & Mapped reads & $\begin{array}{c}\text { Mapping } \\
\text { rate (\%) }\end{array}$ \\
\hline Hill & Cys0 & 1 & $11,098,852$ & $8,706,654$ & 79.0 \\
& Cys0 & 2 & $7,432,161$ & $5,528,821$ & 74.4 \\
& Cys0 & 3 & $14,191,549$ & $11,331,100$ & 79.8 \\
& Cys0 & 4 & $10,107,230$ & $8,049,452$ & 79.6 \\
Hill & Cys100 & 1 & $11,326,661$ & $8,739,394$ & 77.2 \\
& Cys100 & 2 & $15,881,554$ & $12,726,762$ & 80.1 \\
& Cys100 & 3 & $12,195,634$ & $9,807,213$ & 80.4 \\
& Cys100 & 4 & $13,405,438$ & $10,636,789$ & 79.3 \\
Z31 & Cys0 & 1 & $24,789,278$ & $18,229,005$ & 73.5 \\
& Cys0 & 2 & $22,532,778$ & $16,268,201$ & 72.2 \\
& Cys0 & 3 & $21,844,814$ & $16,133,350$ & 73.9 \\
& Cys0 & 4 & $21,579,742$ & $15,987,483$ & 74.1 \\
Z31 & Cys100 & 1 & $20,119,176$ & $14,818,590$ & 73.7 \\
& Cys100 & 2 & $21,478,014$ & $16,045,958$ & 74.7 \\
& Cys100 & 3 & $24,494,505$ & $17,793,224$ & 72.6 \\
& Cys100 & 4 & $23,553,167$ & $17,615,606$ & 74.6
\end{tabular}

metabolic activity in plant cells, protecting them against bacterial invasion.

The common down-regulated genes were divided into the following categories: membrane metabolism; extracellular region; hydrolase activity; acting on ester bonds; hydrolase activity; hydrolyzing O-glycosyl compounds; transporter activity; transmembrane transport; carbohydrate metabolic process; and sexual reproduction process. The down-regulated genes were mainly related to membrane metabolism, with $\sim 45 \%$ of the shared DEGs occupying this category (Figure 5). Additionally, $12 \%$ of the down-regulated genes were related to the transmembrane transport category. These results indicated that cysteine might improve the infection efficiency by modifying the cell wall membrane.

\section{Genes Involved in Infection Efficiency Improvement} Genes Involved in the Oxidation-Reduction Process

Among the common up-regulated genes, many are involved in the oxidation-reduction process or oxidoreductase activity process and metabolic process (Table 3). Cysteine synthase 


\section{A}

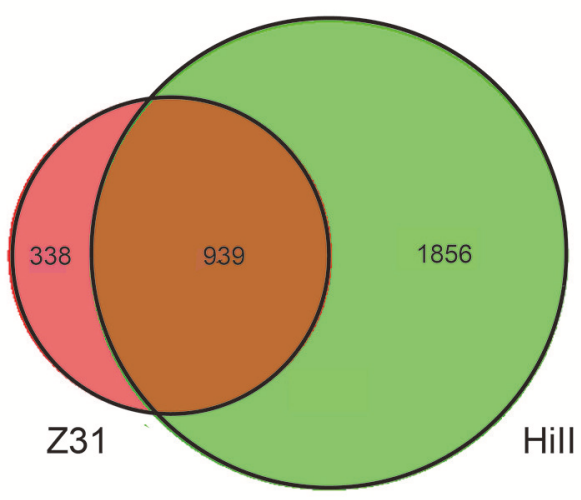

B Down-regulation

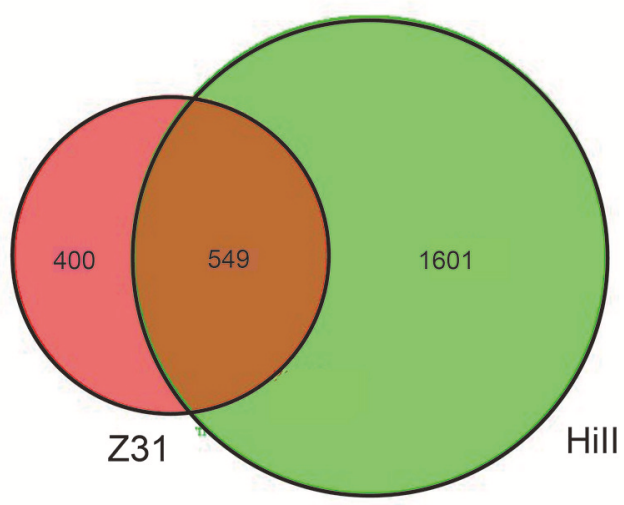

FIGURE 4 | Venn diagram of transcriptome between maize hybrid Hill and inbred line Z31. (A) Shared and unique up-regulated DEGs in Hill and Z31 maize lines. (B) Shared and unique down-regulated DEGs in Hill and Z31 maize lines.

TABLE 2 | Confirmation of the transcriptome results by qRT-PCR.

\begin{tabular}{llcc}
\hline \multirow{2}{*}{ Gene } & Gene annotation & \multicolumn{2}{c}{ Fold change } \\
\cline { 3 - 4 } & & RNA-Seq & qRT-PCR \\
\hline GRMZM2G036708 & Cysteine synthase & 6.44 & 14.22 \\
GRMZM2G170017 & Carbonyl reductase 1 & 13.40 & 19.97 \\
GRMZM2G087875 & Cytochrome P450 CYP81A1 & 25.03 & 31.44 \\
GRMZM2G132875 & NAD(P)H-dependent & 8.84 & 4.90 \\
& oxidoreductase & & \\
GRMZM2G074743 & Alternative oxidase & 18.04 & 6.99 \\
GRMZM2G443445 & Mannitol dehydrogenase & 6.18 & 9.23 \\
GRMZM2G097641 & Sucrose-phosphatase 2 & 7.04 & 8.30 \\
GRMZM2G070322 & Systemin receptor SR160 & 2.66 & 2.27 \\
GRMZM2G025105 & Polygalacturonase inhibitor & 5.46 & 2.60 \\
GRMZM2G166944 & Xyloglucan endotransglucosylase/ & 3.01 & 1.57 \\
& hydrolase protein 23 & & \\
GRMZM2G025190 & Glutathione S-transferase GSTU6 & 14.84 & 7.84 \\
\hline
\end{tabular}

(GRMZM2G036708) is responsible for the final step in biosynthesis of cysteine, catalyzing O3-acetyl-L-serine into L-cysteine. Cysteine synthase also participates in the selenoamino acid metabolism and sulfur metabolism. It has been shown that overexpression of cysteine synthase in transgenic tobacco can increase tolerance to sulfur dioxide and sulfur (Noji et al., 2001).

Superoxide dismutase (SOD) can catalyze the conversion of superoxide radicals to hydrogen peroxide and molecular oxygen, playing a key role in the protection of cell injury induced by oxygen free radicals. The expression of SOD (GRMZM2G169890 and GRMZM2G058522) in maize immature embryos cultured on the medium with cysteine was up-regulated (Table 3), compared with those without cysteine treatment. The addition of cysteine to the maize cultures after Agrobacterium infection could help to maintain the cell health in immature embryos, thus increasing transformation efficiency.
Glycosyltransferase is a transfer enzyme involved in glycosylation, playing important roles in the environmental adaptation of plants. The upregulation of several glycosyltransferase genes (GRMZM2G120016, GRMZM2G052571, and GRMZM5G834303) was seen in maize embryos (Table 3). By up-regulating the expression of these transfer enzymes, the immature embryo cells may be more vigorous after Agrobacterium infection, thereby improving the infection efficiency.

Genes Involved in Membrane Integrity and Transport Many shared down-regulated genes involved in transmembrane integrity and transport were found in the RNA-seq analysis. These genes were primarily members of the aquaporin family (GRMZM2G041980, GRMZM2G392975, GRMZM2G126582, GRMZM2G081843, GRMZM2G027098, GRMZM2G047368, GRMZM2G178693, GRMZM2G154628, and GRMZM2G060922; Table 3). Aquaporins are small membrane proteins that consist of six membrane-spanning $\alpha$-helices connected by five loops (A to E) where the $\mathrm{N}$ and C termini face the cytosol (Murata et al., 2000). The downregulation of these genes may change the permeability of the cell membrane.

\section{Genes Involved in the Cell Wall Metabolism}

The cell wall is the first line of protection defending against the invasion of pathogenic bacteria. Several genes related to the cell wall were differentially expressed in the maize embryos grown on medium containing cysteine. A total of 19 up-regulated DEGs and 40 down-regulated DEGs shared between HiII and Z31 maize lines were identified and mapped to be putatively involved in cell wall metabolism. Among the 19 up-regulated genes, four genes (GRMZM2G166944, GRMZM2G392125, GRMZM2G070271, and GRMZM2G026980) were xyloglucan endotransglycosylase/hydrolase (XTH)-related genes, one gene (GRMZM2G070322) was a hormone receptor protein gene, while the remainder were glycosyltransferases or isomerase. Among 


\section{Up-regulated genes}

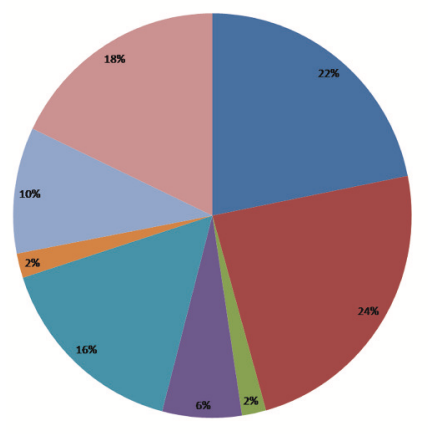

- Metabolic process

- Oxidation-reduction process

- L-phenyalanine catabolic process

- Transferase activity, transferring hexosyl groups

- Oxidoreductase activity

- Ammonia-lyase activity

- Iron ion binding

- Catalytic activity

\section{Down-regulated genes}
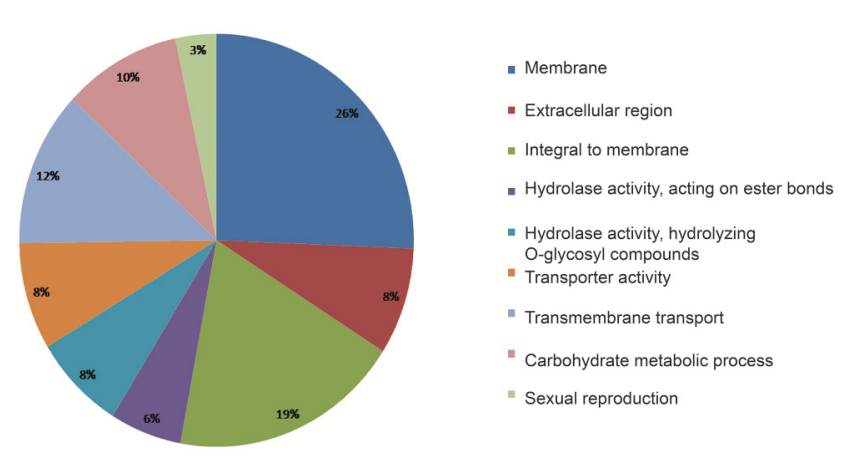

FIGURE 5 | GO analysis of the shared up-regulated (left) and down-regulated (right) DEGs in Hill and Z31 maize embryos.

the 40 down-regulated genes, 10 genes (GRMZM2G021621, GRMZM2G094990, GRMZM2G414779, GRMZM2G339122, GRMZM2G368886, GRMZM2G148485, GRMZM2G082520, GRMZM2G013002, GRMZM2G342246, GRMZM2G021427) were expansin-related genes and five genes (GRMZM2G025231, GRMZM2G178025, GRMZM2G453565, GRMZM2G147687, GRMZM2G141911) were cellulose synthase-related genes (Table 3).

\section{DISCUSSION}

Establishing a high efficient maize transformation system is necessary for the investigation of gene function and development of commercial transgenic maize events. Antioxidants such as dithiothreitol (DTT) or cysteine added into co-cultivation medium has been shown to significantly improve maize transformation efficiency (Frame et al., 2002; Vega et al., 2008). In this study, we confirmed that cysteine could improve the Agrobacterium infection efficiency of inbred line Z31 and hybrid line HiII. We also showed that HiII maize embryos were more sensitive to Agrobacterium infection compared to Z31 embryos (Figure 1). Many reports have demonstrated that HilI can achieve high transformation efficiency (Frame et al., 2002; Vega et al., 2008). Inbred line Z31 is an elite inbred line in China, and its transformation efficiency is relatively lower than HiII (data not shown).

It was proposed that cysteine may act as an antioxidant to minimize cell death caused by Agrobacterium infection (Frame et al., 2002; Vega et al., 2008). To our surprise, the addition of cysteine in the co-cultivation medium significantly increased the accumulation of $\mathrm{H}_{2} \mathrm{O}_{2}$ and $\mathrm{O}_{2}^{-}$(Figure 3), indicating that cysteine may improve the Agrobacterium infection efficiency not just as an antioxidant, but with other mechanism. It has also been shown that high cysteine concentration decreased the proportion of embryos that can give rise to embryogenic callus (Frame et al., 2002; Vega et al., 2008). To investigate the mechanism underlying cysteine-influenced improved Agrobacterium infection efficiency, we performed transcriptome analysis on maize embryos. The sequencing depth for HiII was lower than that for Z31, whereas more reads of HiII were mapped to the B73 reference genome compared to Z31 (Table 1). The HiII is $\mathrm{F}_{2}$ embryos derived from an $F_{1}$ plant originating from a cross between HilI parent $A$ and HilI parent $B$, both progenitors resulting from a cross between inbred lines A188 and B73 (Armstrong et al., 1991). Therefore, it was reasonable that more reads of HiII were mapped to the B73 reference genome than from Z31. Transcriptome profiling analysis showed that the cysteine treatment led to more upregulated and down-regulated genes in HiII embryos than DEGs in Z31 embryos, confirming that HiII was more sensitive to the cysteine treatment than Z31. Numerous shared up-regulated and down-regulated genes were observed in HiII and Z31 embryos cultured on medium with cysteine, indicating that the cysteine treatment had a huge effect on the embryo cell metabolism, thus improving the efficiency of Agrobacterium infection. We also performed transcriptome analysis on the maize embryos treated with heat and found that few genes had changed expression levels compared to the untreated samples (data not shown), indicating that heat treatment might only induce cell competent prior to transformation.

The interaction between Agrobacterium and plant cells during Agrobacterium infection induces major changes in plant expression. Agrobacterium infection upregulated the expression of some plant genes, but also inhibited the expression of some host defense genes. In Arabidopsis tissues infected by Agrobacterium, the DEGs are mainly involved in photosynthesis, carbohydrate metabolism, cell wall synthesis, carbon and nitrogen metabolism, etc. (Deeken et al., 2006). In this study, our purpose was to investigate the mechanism behind cysteineexposed infection efficiency improvement in maize embryos. The cysteine treatment up-regulated 939 genes and down-regulated 549 genes in both Z31 and HiII, respectively (Figure 4). The upregulated genes were mainly involved in the oxidation reduction process, whereas the down-regulated genes were mainly involved in cell wall metabolism.

Plants will have an oxidative burst with high ROS accumulation upon pathogen attack, resulting in programmed cell death (PCD) and cellular defense response (Heller and Tudzynski, 2011; O’brien et al., 2012). To detoxify the oxidative 
TABLE 3 | DEGs which involved in the cell wall metabolism.

\begin{tabular}{|c|c|c|c|}
\hline Pathway & Gene ID & Gene anotation & $\begin{array}{l}\text { Expression } \\
\text { pattern }\end{array}$ \\
\hline & GRMZM2G052571 & $\begin{array}{l}\text { Glutathione } \\
\text { S-transferase }\end{array}$ & Up-regulated \\
\hline & GRMZM2G056388 & $\begin{array}{l}\text { Glutathione } \\
\text { S-transferase }\end{array}$ & Up-regulated \\
\hline \multirow{19}{*}{$\begin{array}{l}\text { Oxidation } \\
\text { reduction } \\
\text { process }\end{array}$} & GRMZM2G036708 & Cysteine synthase & Up-regulated \\
\hline & GRMZM2G159587 & Glyoxylate reductase & Up-regulated \\
\hline & GRMZM2G170017 & Carbonyl reductase 1 & Up-regulated \\
\hline & GRMZM2G177077 & $\begin{array}{l}\text { Glucose-6- } \\
\text { phosphate } \\
\text { 1-dehydrogenase }\end{array}$ & Up-regulated \\
\hline & GRMZM2G141473 & Aldehyde oxidase-2 & Up-regulated \\
\hline & GRMZM2G169890 & $\begin{array}{l}\text { Superoxide } \\
\text { dismutase }\end{array}$ & Up-regulated \\
\hline & GRMZM2G058522 & $\begin{array}{l}\text { Superoxide } \\
\text { dismutase }\end{array}$ & Up-regulated \\
\hline & GRMZM2G471357 & Peroxidase 52 & Up-regulated \\
\hline & GRMZM2G440208 & $\begin{array}{l}\text { 6-phosphogluconate } \\
\text { dehydrogenase }\end{array}$ & Up-regulated \\
\hline & GRMZM2G173195 & $\begin{array}{l}\text { Glycerol-3- } \\
\text { phosphate } \\
\text { dehydrogenase }\end{array}$ & Up-regulated \\
\hline & GRMZM2G090980 & $\begin{array}{l}\text { Mannitol } \\
\text { dehydrogenase }\end{array}$ & Up-regulated \\
\hline & GRMZM2G058244 & $\begin{array}{l}\text { UDP-glucose } \\
\text { 6-dehydrogenase }\end{array}$ & Up-regulated \\
\hline & GRMZM2G053720 & Proline oxidase & Up-regulated \\
\hline & GRMZM2G074743 & Alternative oxidase & Up-regulated \\
\hline & GRMZM2G479423 & Aldose reductase & Up-regulated \\
\hline & GRMZM2G443445 & $\begin{array}{l}\text { Mannitol } \\
\text { dehydrogenase }\end{array}$ & Up-regulated \\
\hline & GRMZM2G099467 & $\begin{array}{l}\text { Gibberellin } 20 \\
\text { oxidase } 2\end{array}$ & Up-regulated \\
\hline & GRMZM2G072529 & Acc oxidase & Up-regulated \\
\hline & GRMZM2G102959 & $\begin{array}{l}\text { Ferredoxin nitrite } \\
\text { reductase }\end{array}$ & Up-regulated \\
\hline \multirow{14}{*}{$\begin{array}{l}\text { Membrane } \\
\text { integrity and } \\
\text { transport }\end{array}$} & GRMZM2G041980 & Aquaporin NIP1-1 & Down-regulated \\
\hline & GRMZM2G392975 & Aquaporin PIP1-1 & Down-regulated \\
\hline & GRMZM2G126582 & Aquaporin NIP-type & Down-regulated \\
\hline & GRMZM2G081843 & Aquaporin PIP 1-3 & Down-regulated \\
\hline & GRMZM2G041980 & Aquaporin NIP1-1 & Down-regulated \\
\hline & GRMZM2G392975 & Aquaporin PIP1-1 & Down-regulated \\
\hline & GRMZM2G126582 & Aquaporin NIP-type & Down-regulated \\
\hline & GRMZM2G081843 & Aquaporin PIP 1-3 & Down-regulated \\
\hline & GRMZM2G027098 & Aquaporin TIP2-2 & Down-regulated \\
\hline & GRMZM2G047368 & Aquaporin PIP2-4 & Down-regulated \\
\hline & GRMZM2G178693 & Aquaporin PIP2-4 & Down-regulated \\
\hline & GRMZM2G154628 & Aquaporin PIP2-4 & Down-regulated \\
\hline & GRMZM2G060922 & Aquaporin SIP1-2 & Down-regulated \\
\hline & GRMZM2G159632 & Sulfate transporter & Down-regulated \\
\hline \multirow{2}{*}{$\begin{array}{l}\text { Membrane } \\
\text { integrity and } \\
\text { transport }\end{array}$} & GRMZM2G442523 & $\begin{array}{l}\text { Sugar transport } \\
\text { protein } 5\end{array}$ & Down-regulated \\
\hline & GRMZM2G063824 & $\begin{array}{l}\text { Carbohydrate } \\
\text { transporter }\end{array}$ & Down-regulated \\
\hline
\end{tabular}

(Continued)
TABLE 3 | Continued

\begin{tabular}{|c|c|c|c|}
\hline Pathway & Gene ID & Gene anotation & $\begin{array}{l}\text { Expression } \\
\text { pattern }\end{array}$ \\
\hline & GRMZM2G342907 & Sulfate transporter & Down-regulated \\
\hline & GRMZM2G036448 & $\begin{array}{l}\text { Amino } \\
\text { acid-polyamine } \\
\text { transporter }\end{array}$ & Down-regulated \\
\hline \multirow[t]{22}{*}{$\begin{array}{l}\text { Cell wall } \\
\text { metabolism }\end{array}$} & GRMZM2G166944 & $\begin{array}{l}\text { Xyloglucan } \\
\text { endotransglucosylase }\end{array}$ & Up-regulated \\
\hline & GRMZM2G392125 & $\begin{array}{l}\text { xyloglucan } \\
\text { endotransglucosylase }\end{array}$ & Up-regulated \\
\hline & GRMZM2G070271 & $\begin{array}{l}\text { Probable xyloglucan } \\
\text { endotransglucosylase }\end{array}$ & Up-regulated \\
\hline & GRMZM2G026980 & $\begin{array}{l}\text { Xyloglucan } \\
\text { endotransglycosylase }\end{array}$ & Up-regulated \\
\hline & GRMZM2G070322 & $\begin{array}{l}\text { Systemin receptor } \\
\text { SR160 }\end{array}$ & Up-regulated \\
\hline & GRMZM2G021621 & Expansin-B4 & Down-regulated \\
\hline & GRMZM2G094990 & Beta-expansin 1a & Down-regulated \\
\hline & GRMZM2G414779 & Expansin-A31-like & Down-regulated \\
\hline & GRMZM2G339122 & Alpha expansin 1 & Down-regulated \\
\hline & GRMZM2G368886 & Alpha expansin 4 & Down-regulated \\
\hline & GRMZM2G148485 & Expansin-B15-like & Down-regulated \\
\hline & GRMZM2G082520 & Beta-expansin 1a & Down-regulated \\
\hline & GRMZM2G013002 & Beta expansin8 & Down-regulated \\
\hline & GRMZM2G342246 & Beta-expansin 7 & Down-regulated \\
\hline & GRMZM2G021427 & Expansin-B3-like & Down-regulated \\
\hline & GRMZM2G025231 & Cellulose synthase 7 & Down-regulated \\
\hline & GRMZM2G178025 & $\begin{array}{l}\text { Endoglucanase } \\
\text { 12-like }\end{array}$ & Down-regulated \\
\hline & GRMZM2G453565 & Endoglucanase 2-like & Down-regulated \\
\hline & GRMZM2G147687 & Exoglucanase 1 & Down-regulated \\
\hline & GRMZM2G141911 & Endoglucanase 4-like & Down-regulated \\
\hline & GRMZM2G131912 & Pectate lyase 8 & down-regulated \\
\hline & GRMZM2G091191 & $\begin{array}{l}\text { Brassinosteroid- } \\
\text { regulated protein } \\
\text { BRU1-like }\end{array}$ & Down-regulated \\
\hline
\end{tabular}

stress, plant cells have several enzymatic and non-enzymatic systems. Among the common up-regulated genes in HiII and Z31 maize embryos, many were involved in the oxidation-reduction process or oxidoreductase activity process and metabolic process (Table 3). Cysteine synthase (GRMZM2G036708), an enzyme responsible for the final step in cysteine biosynthesis, was also upregulated in maize embryos after Agrobacterium infection, indicating that plant cells might need more antioxidants, i.e., cysteine, to cope with the oxidative stress. SOD can catalyze the conversion of superoxide radicals to hydrogen peroxide and molecular oxygen, playing a key role in the protection of cell injury induced by oxygen free radicals. The expression of SOD (GRMZM2G169890 and GRMZM2G058522) in maize immature embryos cultured on the medium with cysteine was up-regulated (Table 3) compared to those without cysteine treatment. This may be helpful to maintain the cellular homeostasis of immature embryos, increasing the 
efficiency of Agrobacterium infection. The up-regulation of several glycosyltransferase genes (GRMZM2G120016, GRMZM2G052571, and GRMZM5G834303) in maize embryos was also observed (Table 3), that might participate in the regulation of redox status. Glycosyltransferase is a kind of transfer enzyme involved in glycosylation. It has been shown that several Arabidopsis glycosyltrasferase can regulate the redox status and detoxify the ROS (Simon et al., 2014).

We found that the addition of cysteine in the cocultivation medium significantly decreased cell wall-related protein occurrence. This indicated that the addition of cysteine improved the Agrobacterium infection efficiency not only by increasing expression of detoxifying enzymes but also affecting the expression of genes involved in the cell membrane and cell wall metabolism. Aquaporin proteins are multifunctional and act as a selective channel protein for water passage as well as in the physiological processes of other material transport, cell elongation and differentiation (Chaumont and Tyerman, 2014). Several aquaporin genes were down-regulated in the maize embryos treated with cysteine. The down-regulation of these genes may change the permeability of the cell membrane and increase water channel activity, providing favorable conditions for the invasion of Agrobacterium.

The cell wall is the initial grounds of defense against pathogenic bacteria invasion. Several genes related to the cell wall were up-regulated or down-regulated in maize embryos grown on medium containing cysteine. Xyloglucan endotransglucosylase/hydrolase (XTH) is a cell wall loosening enzyme and plays a key role in relaxing the cell wall (Rose et al., 2002; Van Sandt et al., 2007). The expression of XTH is significantly increased during the ripening of Kiwifruit (Redgwell and Fry, 1994) and tomato (Miedes and Lorences, 2009), indicating that XTH is involved in cell wall degradation, resulting in ripened fruit that turn soft upon maturity. When overexpressing $\mathrm{ZmXTH1}$ in Arabidopsis, the activity of glucan hydrolase increases and the structure and composition of the cell wall significantly change in transgenic Arabidopsis (Genovesi et al., 2008). In this study, the up-regulated expression of cell wall integrity-related genes such as, XTH, glucose isomerase, and isomerase may have affected the maize embryo cell wall structure, thus altering the infection efficiency.

Expansins are plant cell wall-loosening proteins and encoded by multigene families in land plants (Javier and Cosgrove, 2005). It was found that the down-regulated expression of expansin gene inhibits the growth and development of plants (Pien et al., 2001). In most cases, the overexpression of expansion genes can stimulate the plant cell growth (Ma et al., 2013). It is proposed that expansion proteins can anchor the surface of cellulose, thus being able to detach glucans from the cellulose surface, whereas it is also possible that expansins can decrease the activity of some cell wall degrading enzymes (Nardi et al., 2013). In this study, the down-regulation of several expansion genes may stimulate the cell wall degradation. Cellulose synthase is a key enzyme in cell wall synthesis and 12 genes have been identified in maize (Appenzeller et al., 2004). It has been shown that mutation of a cellulose synthase-like gene inhibits the Agrobacterium transformation of Arabidopsis root (Zhu et al., 2003a). Together, the change in cellulose synthase and expansin genes induced by the cysteine treatment may lead to the high cell wall elasticity and slow cell proliferation. This would keep the cell wall in a relatively permeable state so as to improve the infection efficiency of the embryos.

\section{CONCLUSION}

The addition of cysteine in the co-cultivation medium significantly increased the Agrobacterium infection efficiency on maize embryos. The addition of cysteine in the cocultivation medium significantly increased the accumulation of ROS in embryos, indicating that cysteine may improve the Agrobacterium infection efficiency not just as an antioxidant. Transcriptome profiling analysis revealed that the addition of cysteine induced up-regulation in genes mainly involved in the oxidation reduction process, whereas the down-regulated genes were mainly involved in cell wall metabolism. We hypothesize that cysteine could loosen the cell wall by modifying cell wall and membrane metabolism during Agrobacterium infection, thereby improving the entry of Agrobacterium into plant cells.

\section{AUTHOR CONTRIBUTIONS}

YL, JW, and YjL designed the research. YL performed the research. ZZ, YL, JF, GW, and YjL analyzed the data. YL, JW, and YjL wrote the article.

\section{FUNDING}

National Major Project for Transgenic Organism Breeding (2016ZX08010-004) and the Agricultural Science and Technology Innovation Program of CAAS.

\section{ACKNOWLEDGMENTS}

This work was supported by National Major Project for Transgenic Organism Breeding (2016ZX08010-004). We are grateful to the reviewers for the valuable advice to improve the manuscript.

\section{AVAILABILITY OF SUPPORTING DATA}

The sequencing data were deposited in the NCBI Sequencing Read Archive (SRA) with accession number SRR5980095, SRR5980096, SRR5979992, SRR5980097, SRR5980098, SRR5980099, SRR5980100, SRR5980101, SRR5980102, SRR5980105, SRR5980110, SRR5980111, SRR5980112, SRR5980113, SRR5980132, and SRR5980133.

\section{SUPPLEMENTARY MATERIAL}

The Supplementary Material for this article can be found online at: https://www.frontiersin.org/articles/10.3389/fpls.2017. 01778/full\#supplementary-material 


\section{REFERENCES}

Altpeter, F., Springer, N. M., Bartley, L. E., Blechl, A., Brutnell, T. P., Citovsky, V., et al. (2016). Advancing crop transformation in the era of genome editing. Plant Cell 28, 1510-1520. doi: 10.1105/tpc.16.00196

Anand, A., Rojas, C. M., Tang, Y., and Mysore, K. S. (2012). Several components of SKP1/Cullin/F-box E3 ubiquitin ligase complex and associated factors play a role in Agrobacterium-mediated plant transformation. New Phytol. 195, 203-216. doi: 10.1111/j.1469-8137.2012. 04133.x

Appenzeller, L., Doblin, M., Barreiro, R., Wang, H., Niu, X., Kollipara, K., et al. (2004). Cellulose synthesis in maize: isolation and expression analysis of the cellulose synthase (CesA) gene family. Cellulose 11, 287-299. doi: 10.1023/B:CELL.0000046417.84715.27

Armstrong, C. L., Green, C. E., and Phillips, R. L. (1991). Development and availability of germplasm with high Type II culture formation response. Maize Genet. Coop. News Lett. 65, 92-93.

Brencic, A., and Winans, S. C. (2005). Detection of and response to signals involved in host-microbe interactions by plant-associated bacteria. Microbiol. Mol. Biol. Rev. 69, 155-194. doi: 10.1128/MMBR.69.1.155-194.2005

Chaumont, F., and Tyerman, S. D. (2014). Aquaporins: highly regulated channels controlling plant water relations. Plant Physiol. 164, 1600-1618. doi: 10.1104/pp.113.233791

Cho, H., and Winans, S. C. (2005). VirA and VirG activate the Ti plasmid repABC operon, elevating plasmid copy number in response to woundreleased chemical signals. Proc. Natl. Acad. Sci. U.S.A. 102, 14843-14848. doi: 10.1073/pnas.0503458102

Cho, M. J., Wu, E., Kwan, J., Yu, M., Banh, J., Linn, W., et al. (2014). Agrobactrium-mediated high-frequency transformation of an elite commercial maize (Zea mays L.) inbred line. Plant Cell Rep. 33, 1767-1777. doi: 10.1007/s00299-014-1656-x

Dan, Y., Zhang, S., Zhong, H., Yi, H., and Sainz, M. B. (2015). Novel compounds that enhance Agrobacterium-mediated plant transformation by mitigating oxidative stress. Plant Cell Rep. 34, 291-309. doi: 10.1007/s00299-014-1707-3

Deeken, R., Engelmann, J. C., Efetova, M., Czirjak, T., Muller, T., Kaiser, W. M., et al. (2006). An integrated view of gene expression and solute profiles of Arabidopsis tumors: a genome-wide approach. Plant Cell 18, 3617-3634. doi: $10.1105 /$ tpc. 106.044743

Djamei, A., Pitzschke, A., Nakagami, H., Rajh, I., and Hirt, H. (2007). Trojan horse strategy in Agrobacterium transformation: abusing MAPK defense signaling. Science 318, 453-456. doi: 10.1126/science. 1148110

Dutilleul, C., Garmier, M., Noctor, G., Mathieu, C., Chetrit, P., Foyer, C. H., et al. (2003). Leaf mitochondria modulate whole cell redox homeostasis, set antioxidant capacity and determine stress resistance through altered signaling and dirunal regulation. Plant Cell 15, 1212-1226. doi: 10.1105/tpc.009464

Enríquez-Obregón, G. A., Vázquez-Padrón, R. I., Prieto-Samsonov, D. L., Riva, G. A. D. L., and Selman-Housein, G. (1998). Herbicide-resistant sugarcane (Saccharum officinarum L.) plants by Agrobacterium-mediated transformation. Planta 206, 20-27. doi: 10.1007/s004250050369

Frame, B. R., Shou, H., Chikwamba, R. K., Zhang, Z., Xiang, C., Fonger, T. M., et al. (2002). Agrobacterium tumefaciens-mediated transformation of maize embryos using a standard binary vector system. Plant Physiol. 129, 13-22. doi: $10.1104 /$ pp. 000653

Gaspar, Y. M., Nam, J., Schultz, C. J., Lee, L.-Y., Gilson, P. R., Gelvin, S. B., et al. (2004). Characterization of the Arabidopsis lysine-rich arabinogalactan-protein AtAGP17 mutant (rat 1) that results in a decreased efficiency of agrobacterium transformation. Plant Physiol. 135, 2162-2171. doi: 10.1104/pp.104.045542

Gelvin, S. B. (2010). Plant proteins involved in Agrobacteriummediated genetic transformation. Аnnu. Rev. Phytopathol. 48, 45-68. doi: 10.1146/annurev-phyto-080508-081852

Genovesi, V., Fornalé, S., Fry, S. C., Ruel, K., Ferrer, P., Encina, A., et al. (2008). ZmXTH1, a new xyloglucan endotransglucosylase/hydrolase in maize, affects cell wall structure and composition in Arabidopsis thaliana. J. Exp. Bot. 59, 875-889. doi: 10.1093/jxb/ern013

Heller, J., and Tudzynski, P. (2011). Reactive oxygen species in phytopathogenic fungi: signaling, development, and disease. Annu. Rev. Phytopathol. 49, 369-390. doi: 10.1146/annurev-phyto-072910-095355
Hiei, Y., Ishida, Y., Kasaoka, K., and Komari, T. (2006). Improved frequency of transformation in rice and maize by treatment of immature embryos with centrifugation and heat prior to infection with Agrobacterium tumefaciens. Plant Cell Tissue Org. Cult. 87, 233-243. doi: 10.1007/s11240-006-9 157-4

Ishida, Y., Saito, H., Ohta, S., Hiei, Y., Komari, T., and Kumashiro, T. (1996). High efficiency transformation of maize (Zea mays L.) mediated by Agrobacterium tumefaciens. Nat. Biotechnol. 14, 745-750. doi: 10.1038/nbt0696-745

Javier, S., and Cosgrove, D. J. (2005). The expansin superfamily. Genome Biol. 6:242. doi: 10.1186/gb-2005-6-12-242

Jefferson, R. A., Kavanagh, T. A., and Bevan, M. W. (1987). GUS fusions: betaglucuronidase as a sensitive and versatile gene fusion marker in higher plants. ЕМВО J. 6, 3901-3907.

Lai, E. M., Shih, H. W., Wen, S. R., Cheng, M. W., Hwang, H. H., and Chiu, S. H. (2006). Proteomic analysis of Agrobacterium tumefaciens response to the Vir gene inducer acetosyringone. Proteomics 6, 4130-4136. doi: 10.1002/pmic.200600254

Livak, K. J., and Schmittgen, T. D. (2001). Analysis of relative gene expression data using real-time quantitative PCR and the $2^{-\Delta \Delta \mathrm{C}_{(\mathrm{T})}}$ method. Methods 25 , 402-408. doi: 10.1006/meth.2001.1262

Lowe, K., Wu, E., Wang, N., Hoerster, G., Hastings, C., Cho, M. J., et al. (2016). Morphogenic regulators baby boom and wuschel improve monocot transformation. Plant Cell 28, 1998-2015. doi: 10.1105/tpc.16.00124

Ma, N., Wang, Y., Qiu, S., Kang, Z., Che, S., Wang, G., et al. (2013). Overexpression of OsEXPA8, a root-specific gene, improves rice growth and root system architecture by facilitating cell extension. PLoS ONE 8:e75997. doi: 10.1371/journal.pone.0075997

Miedes, E., and Lorences, E. P. (2009). Xyloglucan endotransglucosylase/hydrolases (XTHs) during tomato fruit growth and ripening. J. Plant Physiol. 166, 489-498. doi: 10.1016/j.jplph.2008.07.003

Mookkan, M., Nelsonvasilchik, K., Hague, J., Zhang, Z. J., and Kausch, A. P. (2017). Selectable marker independent transformation of recalcitrant maize inbred B73 and sorghum P898012 mediated by morphogenic regulators BABY BOOM and WUSCHEL2. Plant Cell Rep. 36, 1477-1491. doi: 10.1007/s00299-017-2169-1

Murata, K., Mitsuoka, K., Hirai, T., Walz, T., Agre, P., Heymann, J. B., et al. (2000). Structural determinants of water permeation through aquaporin-1. Nature 407:599. doi: 10.1038/35036519

Nardi, C., Escudero, C., Villarreal, N., Martínez, G., and Civello, P. M. (2013). The carbohydrate-binding module of Fragaria $\times$ ananassa expansin 2 (CBMFaExp2) binds to cell wall polysaccharides and decreases cell wall enzyme activities “in vitro." J. Plant Res. 126, 151-159. doi: 10.1007/s10265-012-0504-8

Noji, M., Saito, M., Nakamura, M., Aono, M., Saji, H., and Saito, K. (2001). Cysteine synthase overexpression in tobacco confers tolerance to sulfur-containing environmental pollutants. Plant Physiol. 126, 973-980. doi: $10.1104 /$ pp.126.3.973

O'brien, J. A., Daudi, A., Butt, V. S., and Bolwell, G. P. (2012). Reactive oxygen species and their role in plant defence and cell wall metabolism. Planta 236, 765-779. doi: 10.1007/s00425-012-1696-9

Olhoft, P., and Somers, D. (2001). L -Cysteine increases Agrobacterium -mediated T-DNA delivery into soybean cotyledonary-node cells. Plant Cell Rep. 20, 706-711. doi: 10.1007/s002990100379

Park, S. Y., Yin, X., Duan, K., Gelvin, S. B., and Zhang, Z. J. (2014). Heat shock protein 90.1 plays a role in Agrobacterium-mediated plant transformation. Mol. Plant 7, 1793-1796. doi: 10.1093/mp/ssu091

Pien, S., Wyrzykowska, J., McQueen-Mason, S., Smart, C., and Fleming, A. (2001). Local expression of expansin induces the entire process of leaf development and modifies leaf shape. Proc. Natl. Acad. Sci. U.S.A. 98, 11812-11817. doi: $10.1073 /$ pnas. 191380498

Pitzschke, A., and Hirt, H. (2010). New insights into an old story: Agrobacteriuminduced tumour formation in plants by plant transformation. EMBO J. 29, 1021-1032. doi: 10.1038/emboj.2010.8

Redgwell, R. J., and Fry, S. C. (1994). Xyloglucan endotransglycosylase activity increases during kiwifruit (Actinidia deliciosa) ripening (implications for fruit softening). Plant Physiol. 103, 1399-1406. doi: 10.1104/pp.103. 4.1399

Rose, J. K., Braam, J., Fry, S. C., and Nishitani, K. (2002). The XTH family of enzymes involved in xyloglucan endotransglucosylation and endohydrolysis: 
current perspectives and a new unifying nomenclature. Plant Cell Physiol. 43, 1421-1435. doi: 10.1093/pcp/pcf171

Sardesai, N., Lee, L. Y., Chen, H., Yi, H., Olbricht, G. R., Stirnberg, A., et al. (2014). Cytokinins secreted by Agrobacterium promote transformation by repressing a plant myb transcription factor. Sci. Signal. 6:ra100. doi: 10.1126/scisignal.2004518

Simon, C., Langlois-Meurinne, M., Didierlaurent, L., Chaouch, S., Bellvert, F., Massoud, K., et al. (2014). The secondary metabolism glycosyltransferases UGT73B3 and UGT73B5 are components of redox status in resistance of Arabidopsis to Pseudomonas syringae pv. tomato. Plant Cell Environ. 37, 1114-1129. doi: 10.1111/pce.12221

Tzfira, T., Vaidya, M., and Citovsky, V. (2002). Increasing plant susceptibility to Agrobacterium infection by overexpression of the Arabidopsis nuclear protein VIP1. Proc. Natl. Acad. Sci. U.S.A. 99, 10435-10440. doi: 10.1073/pnas.162304099

Van Sandt, V. S., Suslov, D., Verbelen, J. P., and Vissenberg, K. (2007). Xyloglucan endotransglucosylase activity loosens a plant cell wall. Ann. Bot. 100, 1467-1473. doi: 10.1093/aob/mcm248

Vega, J. M., Yu, W., Kennon, A. R., Chen, X., and Zhang, Z. J. (2008). Improvement of Agrobacterium-mediated transformation in Hi-II maize (Zea mays) using standard binary vectors. Plant Cell Rep. 27, 297-305. doi: $10.1007 /$ s00299-007-0463-z
Zeng, P., Vadnais, D. A., Zhang, Z., and Polacco, J. C. (2004). Refined glufosinate selection in Agrobacterium-mediated transformation of soybean [Glycine $\max$ (L.) Merrill]. Plant Cell Rep. 22, 478-482. doi: 10.1007/s00299-0030712-8

Zhu, Y., Nam, J., Carpita, N. C., Matthysse, A. G., and Gelvin, S. B. (2003a). Agrobacterium-mediated root transformation is inhibited by mutation of an Arabidopsis cellulose synthase-like gene. Plant Physiol. 133, 1000-1010. doi: 10.1104/pp.103.030726

Zhu, Y., Nam, J., Humara, J. M., Mysore, K. S., Lee, L. Y., Cao, H., et al. (2003b). Identification of Arabidopsis rat mutants. Plant Physiol. 132, 494-505. doi: $10.1104 /$ pp. 103.020420

Conflict of Interest Statement: The authors declare that the research was conducted in the absence of any commercial or financial relationships that could be construed as a potential conflict of interest.

Copyright (c) 2017 Liu, Zhang, Fu, Wang, Wang and Liu. This is an open-access article distributed under the terms of the Creative Commons Attribution License (CC $B Y)$. The use, distribution or reproduction in other forums is permitted, provided the original author(s) or licensor are credited and that the original publication in this journal is cited, in accordance with accepted academic practice. No use, distribution or reproduction is permitted which does not comply with these terms. 